\title{
Crystallization of supercooled liquid antimony: A density functional study
}

\author{
M. Ropo, ${ }^{1,2, *}$ J. Akola, ${ }^{1,3}$ and R. O. Jones ${ }^{4}$ \\ ${ }^{1}$ Laboratory of Physics, Tampere University of Technology, P.O. Box 692, FI-33101 Tampere, Finland \\ ${ }^{2}$ COMP Centre of Excellence, Department of Applied Physics, Aalto University, FI-00076 Aalto, Finland \\ ${ }^{3}$ Department of Physics, Norwegian University of Science and Technology, NO-7491 Trondheim, Norway \\ ${ }^{4}$ Peter Grünberg Institut PGI-1 and JARA/HPC, Forschungszentrum Jülich, D-52425 Jülich, Germany
}

(Received 24 August 2017; published 3 November 2017)

\begin{abstract}
Crystallization of liquid antimony has been studied at $600 \mathrm{~K}$ using six density functional/molecular dynamics simulations with up to 882 atoms and three scenarios: one completely disordered sample that did not crystallize even after $570 \mathrm{ps,} \mathrm{four} \mathrm{with} \mathrm{fixed} \mathrm{crystalline} \mathrm{slab} \mathrm{templates,} \mathrm{and} \mathrm{one} \mathrm{with} \mathrm{a} \mathrm{fixed} \mathrm{crystalline} \mathrm{seed.} \mathrm{Crystallization}$ proceeded layer-by-layer in most cases and was rapid $(\sim 36 \mathrm{~m} / \mathrm{s})$ with templates and somewhat slower with the seed. The seed simulation shows an unusual percolation asymmetry where the crystallite grows faster in the direction normal to the zigzag planes. Changes in pair distribution functions, bond angle distributions, ring statistics, nearest-neighbor distances, and cavity volumes were monitored. Diffusion plays a minor role in the process, and the evolution of bond lengths and ring statistics supports the bond-interchange model introduced to explain the rapid crystallization of Sb-rich phase change materials.
\end{abstract}

DOI: 10.1103/PhysRevB.96.184102

\section{INTRODUCTION}

Crystallization of amorphous antimony and tellurium occurs extremely rapidly, and the processes involved have fascinated scientists for over 100 years [1]. Amorphous Te films deposited on substrates crystallize spontaneously when warmed above $285 \mathrm{~K}$ [2], and amorphous Sb crystallizes rapidly [3], even "explosively" [4,5], producing heat that drives the crystallization front. It is not surprising that these elements are components of most commercial phase change materials (PCMs), which are based on the rapid and reversible transition between the amorphous $(a)$ and crystalline $(c)$ forms of nanosized "bits" in a very thin polycrystalline layer. The state of a bit is monitored by measuring the reflectivity or resistivity. Alloys of the form $(\mathrm{GeTe})_{x}\left(\mathrm{Sb}_{2} \mathrm{Te}_{3}\right)_{1-x}(\mathrm{GST})$ are common PCMs [6], as are $\mathrm{Sb} / \mathrm{Te}$ alloys near the eutectic composition $\mathrm{Sb}_{70} \mathrm{Te}_{30}$, often containing $\mathrm{Ag}$ and In (AIST) [7]. The crystallization mechanisms differ in the two families: in GST the formation of the rocksalt structure occurs via nucleation [8], in AIST by growth from the surrounding (polycrystalline) region [7].

Crystallization in $a$-AIST was investigated experimentally and theoretically by Matsunaga et al. [7]. Noting that both amorphous and crystalline structures have three short bonds and three longer bonds (" $3+3$ "), these authors proposed a mechanism for crystallization involving a cascade of small displacements of $\mathrm{Sb}$ atoms accompanied by interchanges of short and long bonds. Such local changes can trigger an avalanche across the network, with $(3+3)$ octahedra becoming aligned with the surrounding crystalline region. If this "bond interchange" process occurs in AIST, it is very likely to apply to pure Sb. Furthermore, recent studies of the crystallization dynamics of $\mathrm{Ge}_{x} \mathrm{Sb}_{100-x}$ films in a narrow concentration range $(7<x<10)$ provided evidence for two growth modes [9]: fast growth for $x<8$, and both fast and and slow growth for $8<x<10$. The fast growth mode

\footnotetext{
*matti.ropo@tut.fi
}

shows an interesting correspondence to the bond-interchange model [9].

Crystallization of supercooled liquid $\mathrm{Sb}$ is the focus of the present density functional (DF)/molecular dynamics (MD) simulations for several samples. Similar simulations of $\mathrm{Sb}$ and GeSb compounds (144 atoms, cubic templates) by Hegedüs and Elliott found metastable cubic phases [10], and simulations of $\mathrm{Sb}$ with an $A 7$ template indicated the initial formation of crystals with an unusual layer structure. Experimental crystallization studies have included the roles of film thickness [11], deposition rate [3], gas pressure [3,12], and temperature $[13,14]$.

In our recent $\mathrm{DF} / \mathrm{MD}$ simulations of liquid and supercooled $\mathrm{Sb}$ and $\mathrm{Sb}_{n}$ clusters (up to $n=14$ ) [15], we summarized earlier work on $\mathrm{Sb}$ and discussed the structural and dynamical properties of the liquid from 600 to $1300 \mathrm{~K}$. As in our work on $a$-Te and Te clusters [16], we compared the results with available experimental work. The dynamical properties (diffusion, viscosity) of liquid $\mathrm{Sb}$ were analyzed using dynamical structure factors and current-current correlation functions, as described in detail in our work on liquid $\mathrm{Bi}$ [17]. We extend these calculations here to the crystallization of $\mathrm{Sb}$ samples with up to 882 atoms in the unit cell, using six simulations with three scenarios: a sample initially without crystalline atoms, four with crystalline slab templates, and one with a small, fixed crystalline nucleus. The structural changes are discussed in terms of pair distribution functions (PDFs) and distributions of bond angles, rings, cavities, and near-neighbor distributions. We view the results in the light of the bond interchange model described above [7]. We outline the computational method used in Sec. II and present and discuss the results in Secs. III and IV.

\section{COMPUTATIONAL METHOD}

The density functional [18] simulations were performed using the CPMD program [19] with Born-Oppenheimer molecular dynamics. There are no constraints on the simulations, such as restricting structures to those defined by particular 
collective variables. The electron-ion interaction was described by norm conserving and scalar-relativistic TroullierMartins pseudopotentials [20], and the kinetic-energy cutoff of the plane-wave basis was $20 \mathrm{Ry}$. We used periodic boundary conditions with a monoclinic simulation cell appropriate for the crystalline structure of $\mathrm{Sb}$ with the corresponding number of atoms in the cell, a single $\vec{k}$ point in the Brillouin zone, and the PBEsol approximation [21] for the exchange-correlation energy functional, with a density cutoff for the calculation of the gradient correction of $1.0 \times 10^{-5}$. The time step was 3.0236 fs (125 a.u.), and the temperatures were controlled with a Nosé-Hoover thermostat [22,23] (frequency $800 \mathrm{~cm}^{-1}$, chain length 4$)$. The atomic density in all cases was the measured density at the melting point $\left(903 \mathrm{~K}, 6.467 \mathrm{~g} / \mathrm{cm}^{3}\right)$ [24]. The use of the density at the melting point for all lower temperatures as well as the fact that the simulations are at constant volume lead inevitably to stress in the samples.

To monitor the crystallization progress, we define an order parameter that is based on the octahedral $(3+3)$ coordination and identifies the coordination of individual $\mathrm{Sb}$ atoms and their coordination polyhedra using a vector quantity ("pseudospin") [7]. For each atom $i$, we define the vector $\vec{S}_{i}$, which contains information about the bond orientation of the atom:

$$
\vec{S}_{i}=\sum_{j} w_{i j} \frac{\overrightarrow{r_{i j}}}{r_{i j}},
$$

where $\overrightarrow{r_{i j}}$ is the vector connecting atoms $i$ and $j, r_{i j}=\left|\overrightarrow{r_{i j}}\right|$ is the corresponding distance, and $w_{i j}$ is the weight function,

$$
w_{i j}=\frac{1}{1+\exp \left[\left(r_{i j}-c\right) / A\right]} .
$$

The constants $A(0.0217)$ and $c(3.25 \AA)$ are chosen so that there is a transition region from 3.0 to $3.5 \AA$ with midpoint $c$. The vector $\vec{S}_{i}$ is then normalized by dividing by its magnitude.

For atom $i$ we write

$$
O_{i}^{\text {atom }}=\sum_{j}\left|\vec{S}_{i} \cdot \vec{S}_{j}\right|,
$$

where $j$ runs over atoms that are less than $3.75 \AA$ from atom $i$. The order parameter for atom $i$ is

$$
O_{i}=\frac{1}{N_{i}+1} \sum_{j} O_{j}^{\mathrm{atom}},
$$

where $j$ runs over the $N_{i}$ nearest neighbors and includes atom $i . O_{i}$ distinguishes locally between the long and short bonds of the $(3+3)$ structure, while incorporating the local environment of atom $i$ and its nearest neighbors. $O_{i}=1$ in perfectly crystalline $\mathrm{Sb}$, and atom $i$ is defined to be "crystalline" if $O_{i}>0.6$.

\section{RESULTS}

\section{A. Supercooled liquid at $600 \mathrm{~K}$}

We attempted to observe crystallization at $600 \mathrm{~K}$ without initial order using a cell with 588 atoms in a starting structure obtained in earlier simulations [15]. Liquid Sb at $1300 \mathrm{~K}$ was cooled to $600 \mathrm{~K}$ in steps of $100 \mathrm{~K}$, with the final steps being $100 \mathrm{ps}$ at $700 \mathrm{~K}$ and $570 \mathrm{ps}$ at $600 \mathrm{~K}$. There were no signs of crystallization at $600 \mathrm{~K}$, and the evolution of the distributions of bond angles, rings, and cavities in 20-ps windows showed only small fluctuations. The mean square displacement (MSD) was a linear function of time, and the average order parameter did not change during the 570-ps trajectory.

Hegedüs and Elliott [10] also observed no signs of crystallization in the absence of a seed or template in their smaller (144 atoms) and shorter (90 ps) simulations. Ronneberger [25] performed a series of DF/MD simulations on liquid Sb samples with 360 atoms in the cell. Crystallization was not observed at temperatures below $300 \mathrm{~K}$ and above $600 \mathrm{~K}$, but occurred at intermediate temperatures in $\sim 50 \mathrm{ps}$. Although simulations with different time and length scales may be difficult to compare, they emphasize the problems facing simulations of crystallization in $\mathrm{Sb}$ at $600 \mathrm{~K}$.

Kalikka et al. [26] found that simulations of $\sim 1$ ns were needed to observe the first clear signs of crystallization in $\mathrm{Ge}_{2} \mathrm{Sb}_{2} \mathrm{Te}_{5}$, although samples with a small, fixed crystalline nucleus ordered at 500, 600, and $700 \mathrm{~K} \mathrm{[27].} \mathrm{The} \mathrm{growth}$ mechanisms differ in Sb and GST, and longer crystallization times might be expected in the more homogeneous samples of $\mathrm{Sb}$. Simulations of this scale might require ordered nuclei or a crystalline template to trigger the process, as we now discuss.

\section{B. Supercooled liquid-crystal interface}

The first template calculations considered a crystalline slab (294 atoms, six atomic layers) in contact with a liquid structure from the $700-\mathrm{K}$ simulation described above (588 atoms, thickness corresponding to 12 crystalline atomic layers). The total number of atoms in this simulation (run 0) was 882 and the cell dimensions were $30.64 \times 30.64 \times 33.92 \AA^{3}$. We performed three smaller simulations (runs 1-3) with 450 atoms including a crystalline template. The height of the unit cell perpendicular to the template ( $z$ direction) was the same $(33.92 \AA)$ as in the cell with 882 atoms, and the starting atomic configurations of the 450-atom simulations were identical. The liquid structure was again taken from the 700-K trajectory used in the larger simulation, but the cell was reduced in the $x y$ plane (parallel to the template, $21.89 \times 21.89 \AA^{2}$ ), and the number of atoms was adjusted accordingly. The crystalline template had six layers, as in run 0 , and the initial velocity distributions were generated for each run corresponding to Maxwell-Boltzmann distributions at $600 \mathrm{~K}$.

The crystallization energetics at $600 \mathrm{~K}, \mathrm{MSD}$, fraction of crystalline atoms, and percolation of the crystalline region are shown in Fig. 1. The simulation times were run 0: $46 \mathrm{ps}$; run 1: $39 \mathrm{ps}$; run 2: $28 \mathrm{ps}$; and run 3: $26 \mathrm{ps}$. The energy curves, especially for run 0 , show spikes at the end of the runs, where much of the cell is crystalline with small pockets of disordered atoms. These features reflect numerical problems in the electronic structure convergence (direct inversion of the iterative subspace) when few atoms are disordered and the $\mathrm{Sb}$ structure becomes semimetallic.

The starting configuration for the template calculations can be seen in the topmost panels of Figs. 2 and 3, where the crystalline template under the disordered region is apparent. The motion of some atoms of the initial crystalline slab in run 0 was sufficient that they no longer satisfied the criterion of crystallinity (see the 2-ps snapshot of Fig. 2). Similar changes 

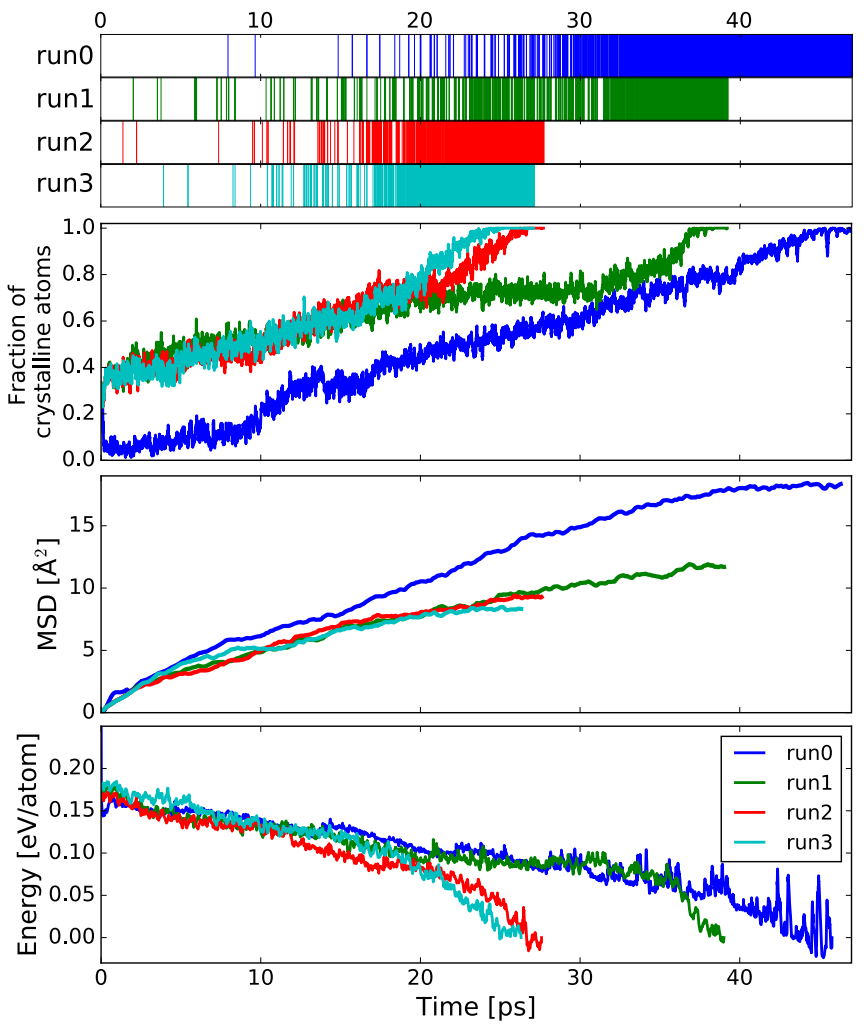

FIG. 1. Crystallization simulations runs $0-3$ at $600 \mathrm{~K}$ : percolation (in the $z$ direction), fraction of crystalline atoms, MSD, and potential energy.

were not found in runs $1-3$, but the labels of some atoms at the crystalline-liquid interfaces changed.

The fraction of crystalline atoms in the second panel of Fig. 1 shows that recrystallization of the partially disordered slab (one-third of the atoms) in run 0 was complete after 11-12 ps, having been triggered by the percolation of recrystallized atoms in the $x y$ plane (parallel to initial crystalline plane). This was followed by a period of relative stability, after which there were approximately six crystalline atomic layers (Fig. 2, 13 ps). Crystallization occurred via layer-by-layer growth in all cases (see Figs. 2 and 3). There was a brief interlude in run 0 near $39 \mathrm{ps,} \mathrm{where} \mathrm{two} \mathrm{disordered} \mathrm{pockets} \mathrm{became}$ connected by disordered atoms, and crystallization continued several ps later. Such events emphasize the stochastic nature of the process and were also found in our studies of GST [26]. In runs $1-3$, layer-by-layer crystallization started after $\sim 2-3 \mathrm{ps,}$ and proceeded steadily throughout run 2 and run 3 . In run 1 , crystallization between 20 and 30 ps was followed by 10 ps with little structural change (see Fig. 1). At this point five to six disordered layers remained.

Crystallization in runs $0-3$ proceeded approximately layer by layer (see Figs. 2 and 3 ) with similar speeds, with the first six layers ordering in $\sim 20 \mathrm{ps}$ in all cases. Complete crystallinity was achieved in run 0: $36 \mathrm{ps}$, runs $1-3: 39,28$, and $27 \mathrm{ps,}$ respectively. The time for run 0 was estimated from the point where the initial crystalline slab recrystallized, as the initial disappearance may be due to fluctuations. The speeds were then estimated by dividing the height of the disordered part $(\sim 22.6 \AA)$ by the crystallization time and noting that there are
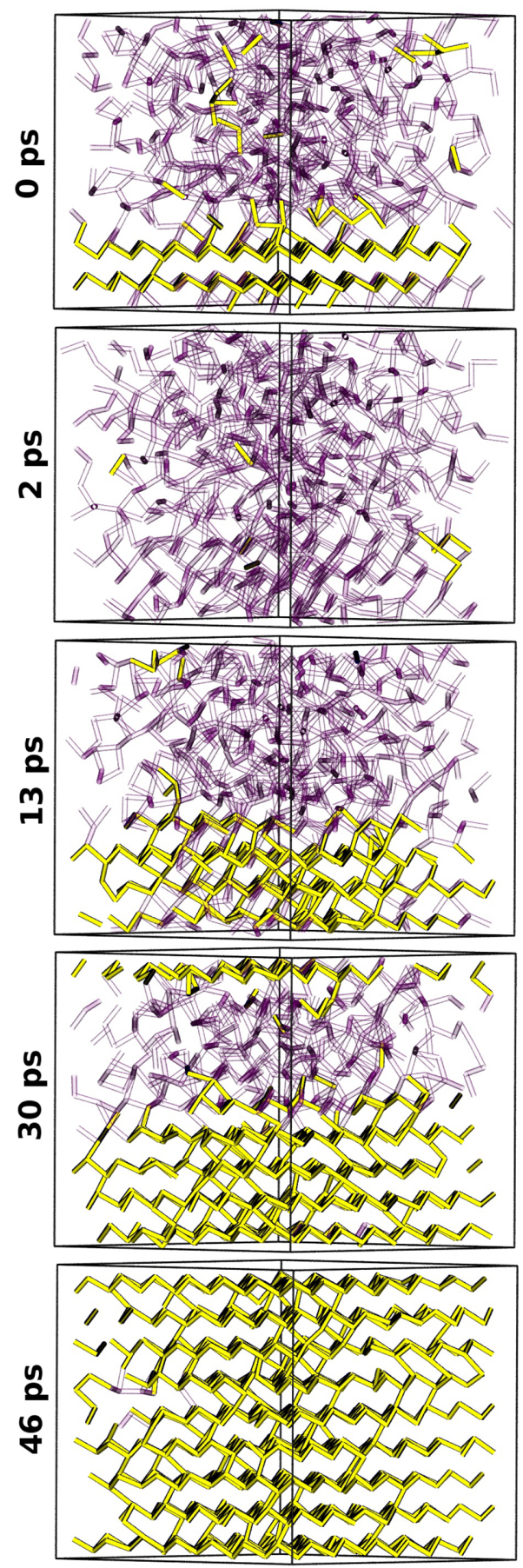

FIG. 2. Large system (882 atoms), run 0: Five snapshots showing crystalline atoms at $0,2,13,30$, and 46 ps. Yellow tubes connect crystalline atoms, transparent purple tubes disordered atoms.

two crystallization fronts. The results are run $0: 32 \mathrm{~m} / \mathrm{s}$; run 1: $29 \mathrm{~m} / \mathrm{s}$; run $2: 41 \mathrm{~m} / \mathrm{s}$; and run $3: 42 \mathrm{~m} / \mathrm{s}$, with an average of $36 \mathrm{~m} / \mathrm{s}$. The slope of the fraction of the crystalline atoms in run 1 and the other smaller samples are the same up to $19 \mathrm{ps}$, and the difference is due to the break in crystallization for $10 \mathrm{ps}$ in run 1 . The calculated speeds are in reasonable agreement with the experimental value found in $\mathrm{Ge}_{7} \mathrm{Sb}_{93}$ at $800 \mathrm{~K}(21 \mathrm{~m} / \mathrm{s})$ [28], where the analysis was based on classical nucleation theory $[28,29]$. The growth rate was found to increase with decreasing Ge concentration. 


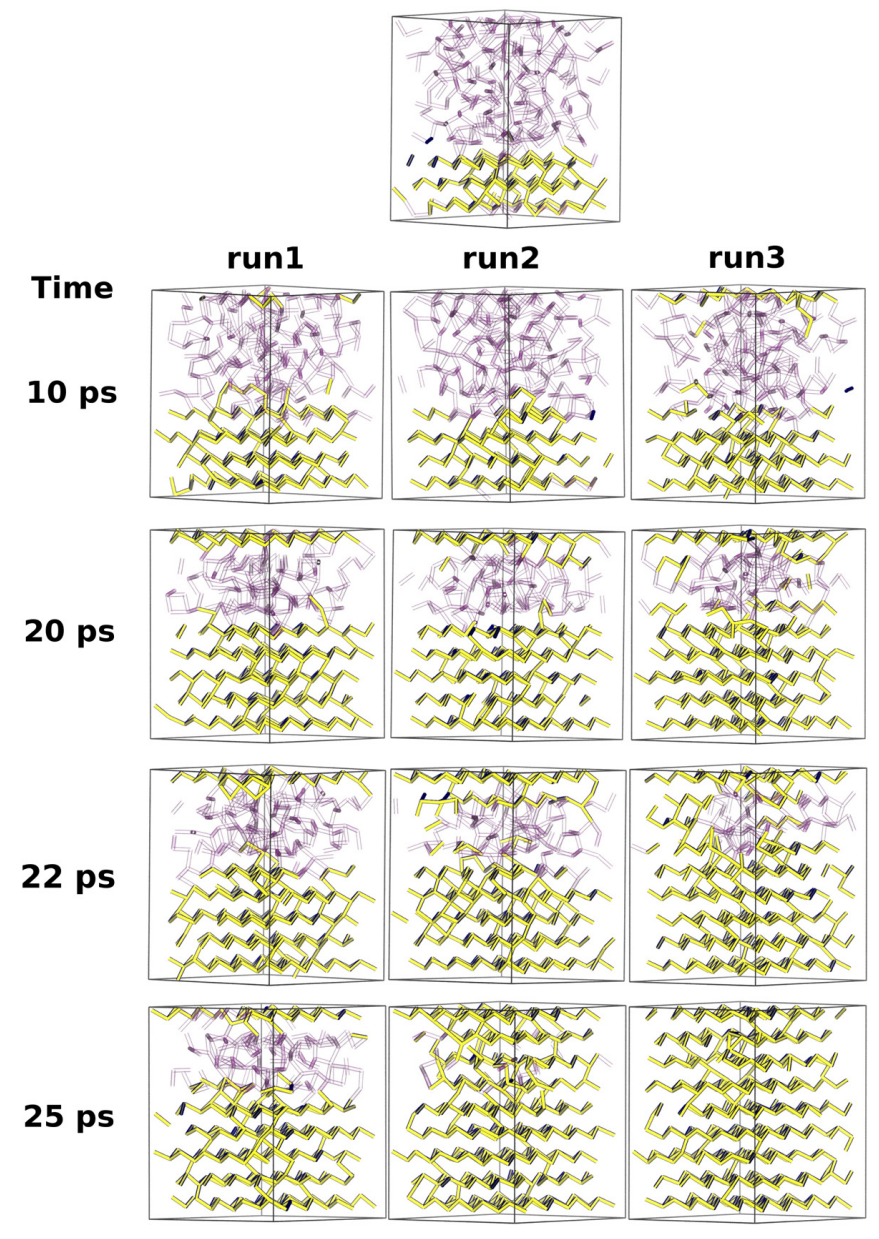

FIG. 3. Smaller systems (450 atoms), run 1-3: Snapshots after 0, $10,20,22$, and 25 ps.

\section{Supercooled liquid with fixed seed}

A crystalline seed of 20 atoms was inserted into a liquid sample at $700 \mathrm{~K}$ and overlapping atoms were removed, leading to 588 atoms in the original hexagonal cell of $30.64 \times 30.64 \times$ $22.61 \AA^{3}$. The seed comprised two zigzag units (four atomic layers) of ten atoms each, approximately cylindrical with respect to the $c$ direction of the seed, the atomic coordinates of which were fixed throughout the simulation (147 ps). The MSD, energy, and percolation of crystal atoms are shown in Fig. 4. Percolation occurred in the $z$ direction (parallel to the $c$ axis of the seed) and in the $x y$ plane, where percolation was not evident initially. The starting configuration is shown in the uppermost panel ( $0 \mathrm{ps})$ of Fig. 5, and the energy curve shows spikes similar to those observed at the end of run 0 .

The crystal seed grew preferably in the $z$ direction (i.e., perpendicular to the zigzag planes of crystalline $\mathrm{Sb}$ ), but growth is slow in the first $100 \mathrm{ps}$, when the nucleus had $\sim 60$ atoms and a diameter of $\sim 11 \AA$. Crystallization was more rapid afterwards, as shown in the second panel of Fig. 4, and percolation in the $z$ direction increased after $\sim 100 \mathrm{ps}$. A further $30 \mathrm{ps}$ were needed to complete the process in the $z$ direction, with the remaining $60 \%$ of the atoms recrystallizing in an additional $17 \mathrm{ps}$.
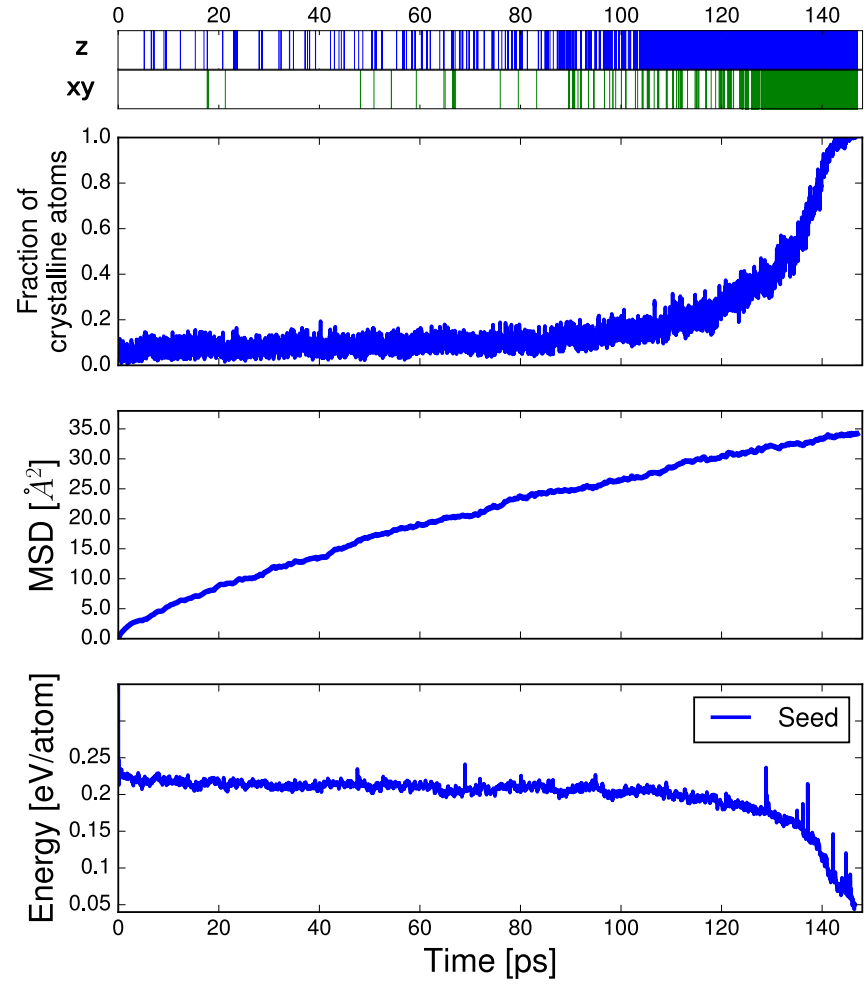

FIG. 4. Simulation with the seed template at $600 \mathrm{~K}$ : Percolation in $z$ and $x y$ planes, fraction of crystalline atoms, MSD, and potential energies.

The crystallization speed was estimated here by comparing the change of the volume to the corresponding change in run 0. Before the critical size was reached $(<100 \mathrm{ps})$, the speed was only $2 \%(0.6 \mathrm{~m} / \mathrm{s})$ of that in the latter, after which it increased to $\sim 70 \%(21 \mathrm{~m} / \mathrm{s})$. The sample percolated in the $z$ direction already at the critical point $(\sim 100 \mathrm{ps})$. The instantaneous speed could be higher: up to $140 \%(45 \mathrm{~m} / \mathrm{s})$ in run 0 between 130 and $140 \mathrm{ps}$. The shape of the simulation cell may have affected the speed at this point.

\section{Structural changes during crystallization}

Further details of the structural changes are now provided for our largest (882 atom) template simulation (run 0) at $600 \mathrm{~K}$. The pair distribution function (PDF) for five windows of $9 \mathrm{ps}$ each are shown in Fig. 6. There is little change in the height of the first peak, but a side peak develops corresponding to the next-nearest neighbors of atoms in a zigzag layer of crystalline $(3+3) \mathrm{Sb}$. As expected, all other peaks became sharper and showed more detail as crystallization proceeded.

The bond angle distributions are shown in Fig. 7 for run 0 at different times, and the cutoff ( $3.75 \AA$ ) was chosen to include all six nearest neighbors. For the initial structure, bond angles have wide peaks at $90^{\circ}$ and $180^{\circ}$ angles and otherwise an almost constant distribution above $50^{\circ}$. During crystallization, the peaks at $90^{\circ}$ and $180^{\circ}$ become even more dominant. Linear configurations $\left(\sim 180^{\circ}\right)$ correspond to the bonds between short and long pairs of nearest neighbors.

Ring distributions during the simulation for the large slab simulation are shown in Fig. 8. They were calculated using 

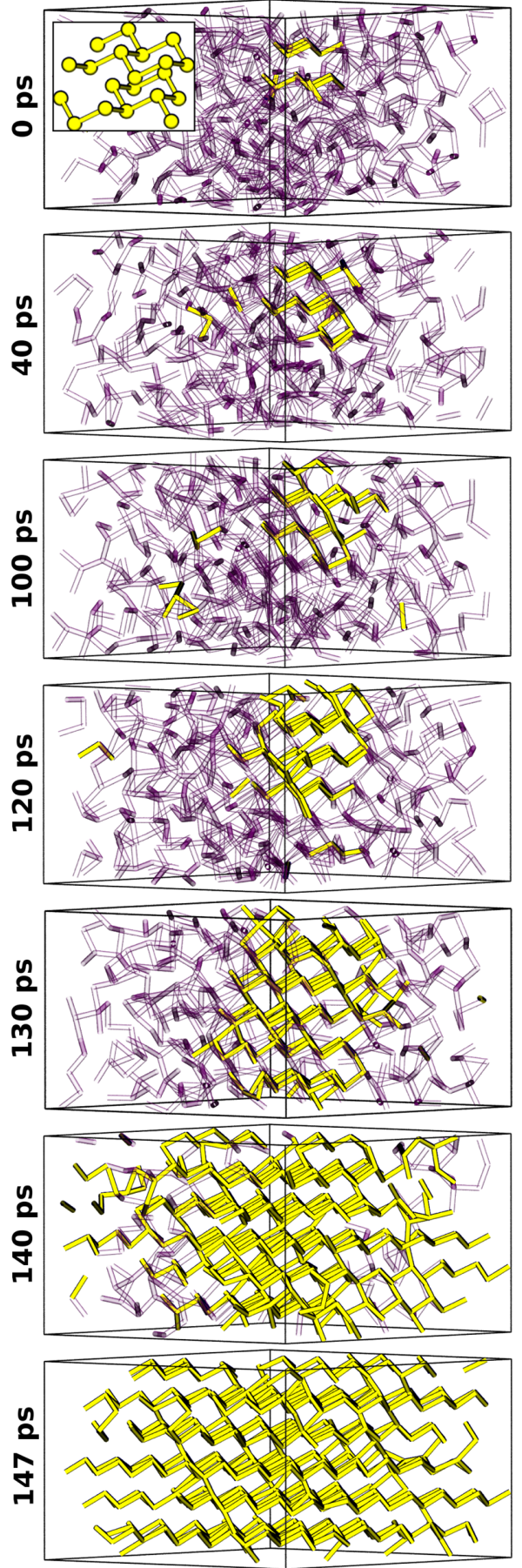

FIG. 5. Simulation with the seed: Snapshots after 0, 40, 100, 120, $130,140,147 \mathrm{ps}$. The seed is shown as an inset in the 0 ps panel.

the ISAACS program [30] with the criterion of Guttman [31] for ring counting and a cutoff determined from the first peak of the pair distribution function $(2.95 \AA)$. Small rings dominate in the initial disordered phase, with numerous fourand fivefold rings. The ring distributions are similar in the first two windows (1-10 ps and 10-19 ps), indicating that partial melting of the initial crystalline slab leads to small changes in the intermediate range order. This contrasts with the bond angle distributions, which differ significantly in the same time windows (see Fig. 7). Finally, crystallization is accompanied

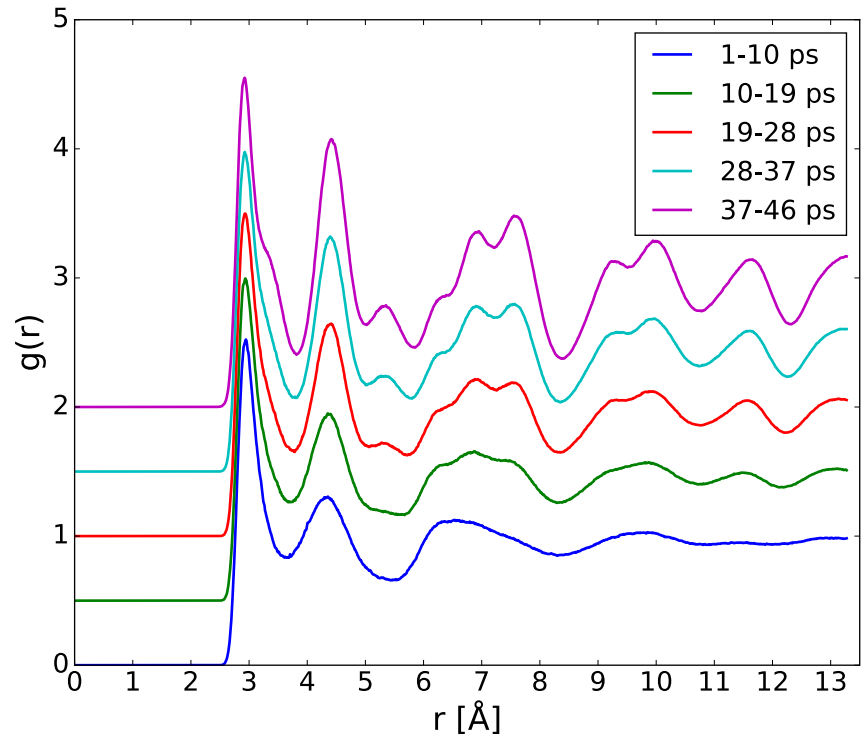

FIG. 6. Evolution of pair distribution function for run 0. Successive curves are shifted by 0.5 .

by the emergence of sixfold rings, which is the only ring type for $c$-Sb.

The distributions of nearest neighbors (Fig. 9) show only small changes for the six nearest neighbors; the first three become closer, as do the next three (corresponding to short and long bonds). The neighbors beyond the sixth, on the other hand, move farther apart as a result of increased order, leading to a gap between the sixth and seventh neighbors. The almost constant distances for the first six neighbors is consistent with the bond-interchange model discussed above, where the main differences between the disordered and crystalline phases are in the orientation of the bonds and in ring statistics.

Cavities were calculated using the PYMOLDYN program [32] with a cutoff radius of $2.6 \AA$ (there were very few cavities with a cutoff of $2.8 \AA$ ). This criterion means that the cavities correspond mainly to the interstitial space between atoms rather than real voids, and even $c$-Sb has "cavities." There is a short period of artificially large cavities at the start of each of

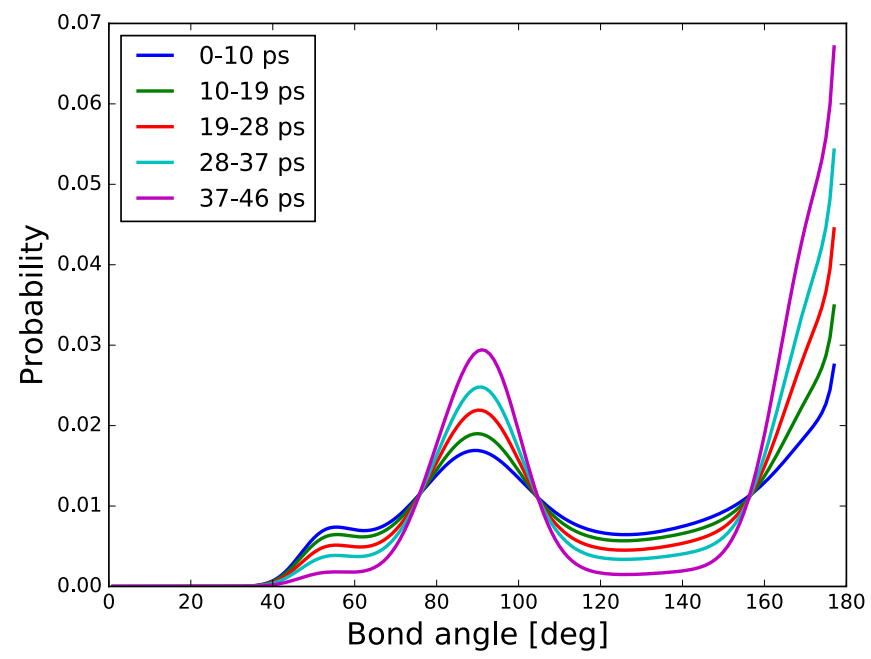

FIG. 7. Evolution of bond angle distribution for run 0 (normalized with $\sin \theta)$. 


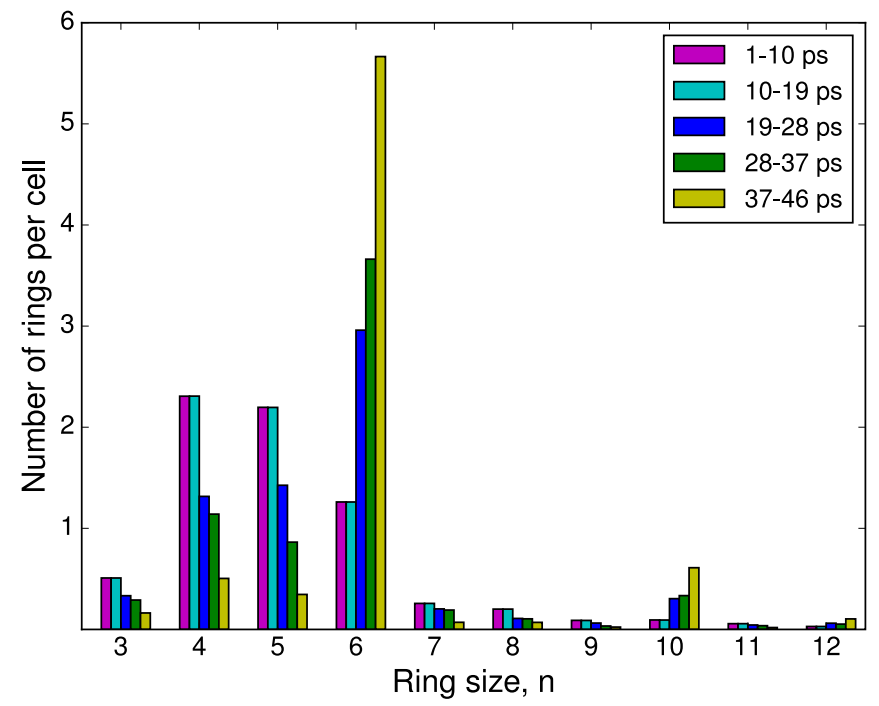

FIG. 8. Variation of ring statistics during run 0 .

runs $0-3$, and afterwards the average cavity volume is $\sim 23 \%$, which is similar to the average volume in the 570-ps simulation at $600 \mathrm{~K}$ (Sec. III A). Crystallization is accompanied by an increase in the cavity volume to $30 \%$ of the total. The cavities in crystalline $\mathrm{Sb}$ are ordered nonoverlapping octahedra between the zigzag planes of the $A 7$ structure. Some octahedra in the disordered phase overlap to form larger cavities, but it is clear that cavities play a smaller role in the crystallization of Sb than in GST [8].

Finally, we studied the diffusion of five atoms during run 0. In Fig. 10, we show the diffusion paths for one atom with average mobility, two with lower mobility, and two that were more mobile than average. Less mobile atoms were located near the interface between the disordered and ordered regions and crystallized quickly, and all five atoms diffused parallel and perpendicular to the crystalline plane. The MSD for each Cartesian direction were similar for the first $15 \mathrm{ps}$, after which diffusion in the $z$ direction was slower than in the $x y$ plane. Diffusion in all three directions ceased only when crystallization neared completion.

The topmost panel of Fig. 1 shows that percolation in the $z$ direction (perpendicular to the crystal template) occurred early in the template runs, in runs 1-3 only a few ps after the start. For run 0 , percolation began during recrystallization (at $\sim 8-10 \mathrm{ps}$ ), but it was more common after 15 ps. The distributions of the six nearest-neighbor distances show little change during crystallization. The ring distributions with major peaks for four- and fivefold rings in the liquid change to sixfold in the crystal, where six-membered rings are prominent. Together with the relatively minor role played by diffusion, these features suggest that the phase change can be understood as a realignment of bonds. This is consistent with the bond-interchange model introduced for AIST by Matsunaga et al. [7], where crystallization is viewed as a rapid succession of diffusionless events that order the $(3+3)$ octahedra along the crystalline $c$ axis. An example of a rapid femtosecond scale event from run 0 is shown in Fig. 11. Each bond interchange changes the coordination of two neighboring atoms that must respond via a backward step or propagation of the bond interchange process.

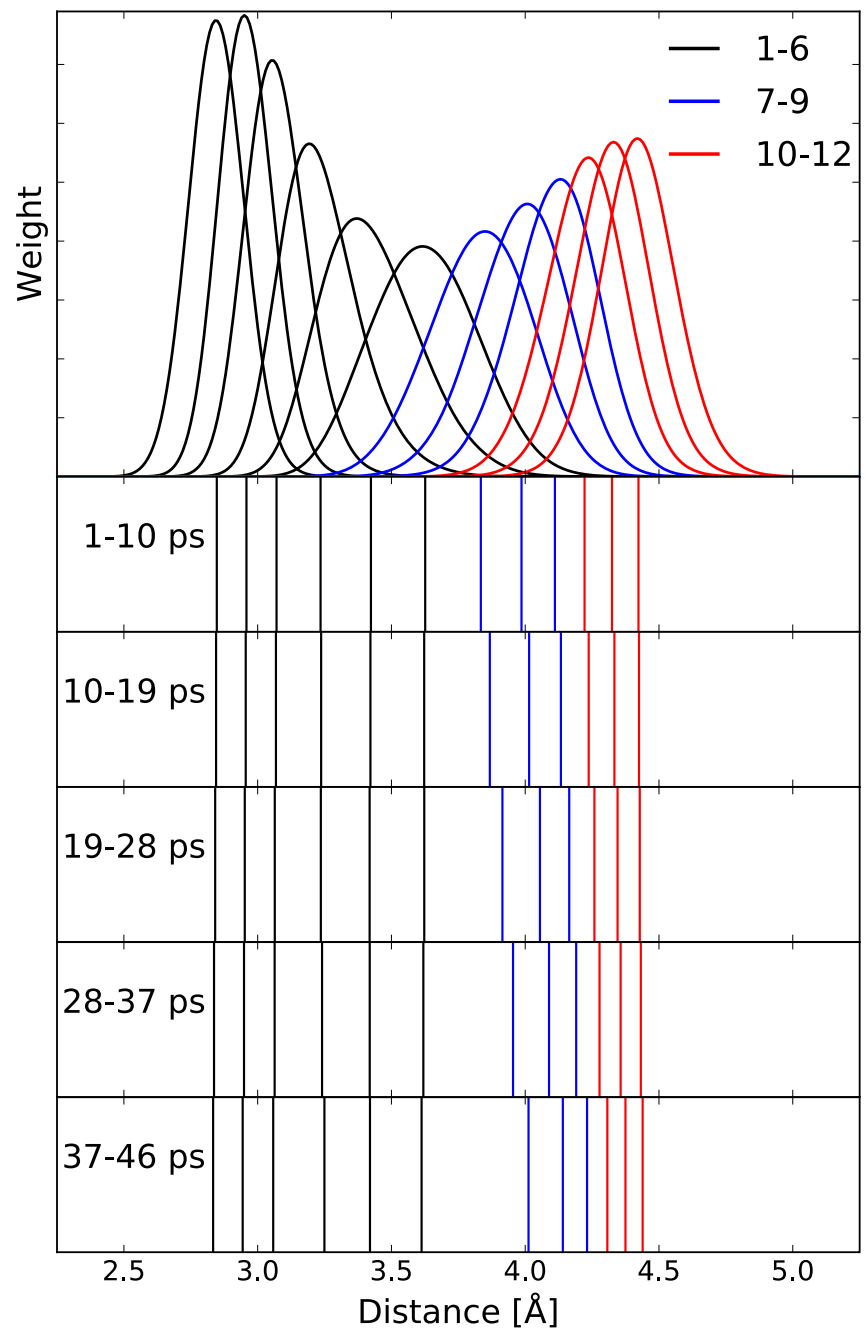

FIG. 9. Near-neighbor distributions for run 0.

\section{E. Comparison with other work}

It is instructive to compare our results with previous DF/MD crystallization studies and experiments on PCM containing $\mathrm{Sb}$. We do this for Sb-dominated materials (Sb, AIST) and for

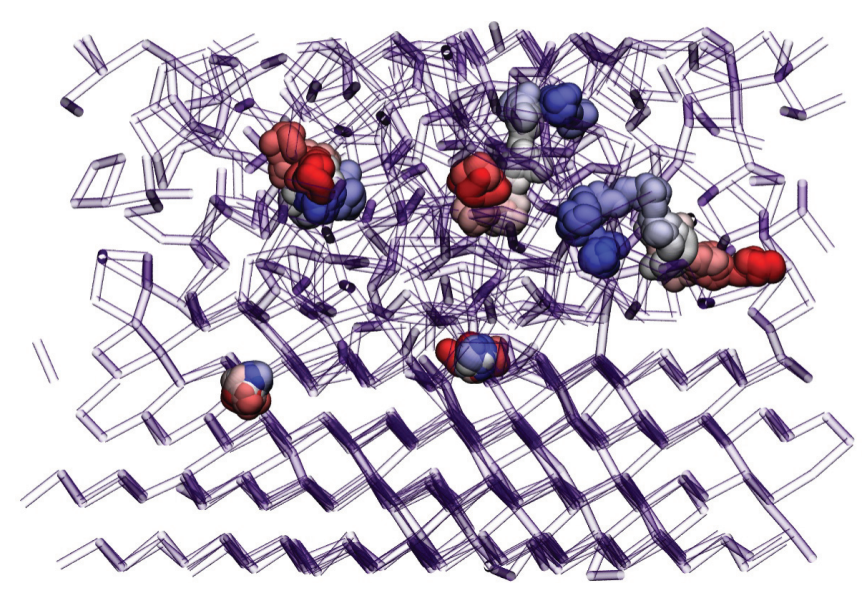

FIG. 10. Simulation run 0: Diffusion of five selected atoms. Red: start; blue: end; purple tubes: connections between atoms after 20 ps. 


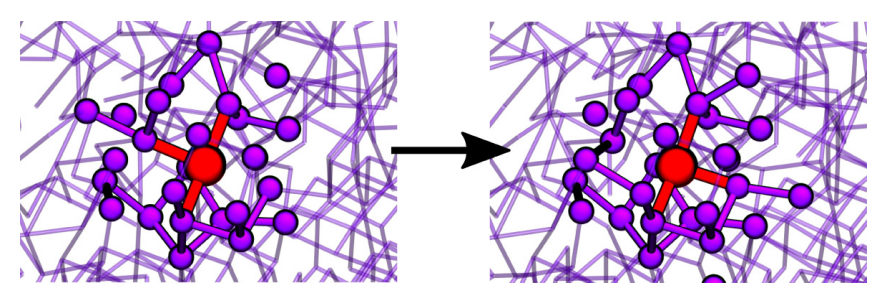

FIG. 11. Example of bond interchange during run 0 . The threefold coordinated center atom switches a short and a long bond. The time between the two frames is $33 \mathrm{fs}$.

$\mathrm{Ge}_{2} \mathrm{Sb}_{2} \mathrm{Te}_{5}$, with particular focus on crystallization speed. In recent DF/MD calculations [26,33], this has been estimated directly from the increase in the number of crystalline atoms.

\section{Sb, AgInSbTe}

Although Hegedüs and Elliott did not provide explicit speeds for their template calculations on $\mathrm{Sb}$ and $\mathrm{Ge}_{15} \mathrm{Sb}_{85}$ [10], their results showed that crystallization takes place at $\sim 2.7 \mathrm{ps}$ /layer (one fixed crystalline layer) and $\sim 4.5 \mathrm{ps}$ /layer (two layers). Our slab template simulations give similar results: $2.99 \mathrm{ps}$ /layer for run 0 (882 atoms), $3.27 \mathrm{ps/layer} \mathrm{for} \mathrm{run} \mathrm{1,}$ $2.31 \mathrm{ps}$ /layer for run 2, and $2.26 \mathrm{ps} /$ layer for run 3 (all 450 atoms). The maximum growth rates measured for template samples of $\mathrm{Ge}_{20} \mathrm{Sb}_{80}, \mathrm{Ge}_{15} \mathrm{Sb}_{85}$, and $\mathrm{Ge}_{10} \mathrm{Sb}_{90}$ are approximately 20, 34, and $58 \mathrm{~m} / \mathrm{s}$ [34], and indicate a higher value for pure $\mathrm{Sb}$. Recent dynamic transition electron microscopy (TEM) measurements provided a direct measurement of laser crystallization in the $\mathrm{Sb}$-rich phase change material $\mathrm{GeSb}_{6} \mathrm{Te}$. The nucleation rates are very low, but the growth rates are up to $10.8 \mathrm{~m} / \mathrm{s}$ for amorphous as-deposited films and significantly higher for an amorphous film subject to pretreatment with subthreshold laser annealing [35]. Both of these results are in reasonable agreement with our findings, given the different compositions and temperatures involved.

Salinga et al. [29] determined the temperature dependence of crystal growth of a film of $\mathrm{Ag}_{4} \mathrm{In}_{3} \mathrm{Sb}_{67} \mathrm{Te}_{26}$ by measuring the time-resolved reflectivity. The crystallization speed varied between $100 \mathrm{~nm} / \mathrm{s}$ at $418 \mathrm{~K}$ and $3.4 \mathrm{~m} / \mathrm{s}$ at $553 \mathrm{~K}$ and obeyed an Arrhenius law over this temperature range. DF/MD simulations of a sample with the same composition (810 atoms with two fixed crystalline layers, 540 atoms without template) have been carried out by Zhang et al. [33], who found a growth velocity of $7.8 \mathrm{~m} / \mathrm{s}$ at $585 \mathrm{~K}$, in reasonable agreement with the measured values. At lower temperatures, particularly at 455 and $500 \mathrm{~K}$, agreement between experiment and simulations is very poor, with the calculated diffusivity being orders of magnitude greater than the measured values. This has been attributed to the much faster quenching rate used to prepare the simulation sample [33], but other factors may contribute.

We have noted that DF/MD simulations of supercooled liquid $\mathrm{Sb}$ [25] did not lead to crystallization at $700 \mathrm{~K}$ and in only one of four samples at $600 \mathrm{~K}$. Simulations at lower temperatures (400-550 K) led to rapid crystallization on a remarkably short time scale ( $\sim 50 \mathrm{ps})$, but no crystallization between 200 and $300 \mathrm{~K}$.

\section{2. $\mathrm{Ge}_{2} \mathrm{Sb}_{2} \mathrm{Te}_{5}$}

Orava et al. [36] measured crystallization in supercooled liquid GST films using ultrafast differential scanning calorimetry between 450 and $650 \mathrm{~K}$, and the growth rate at $650 \mathrm{~K}$ was $2.6 \mathrm{~m} / \mathrm{s}$. Kalikka et al. [8,26] performed DF/MD simulations of 460-atom samples of GST at $600 \mathrm{~K}$ and found speeds of $\sim 0.7-0.9 \mathrm{~m} / \mathrm{s}$ in a sample with a history of crystallization, and $0.3-0.4 \mathrm{~m} / \mathrm{s}$ in samples without. Similar values $(\sim 1 \mathrm{~m} / \mathrm{s})$ were found in DF/MD simulations of GST (460 and 540 atoms, $600 \mathrm{~K}$ ) [37]. Measurements on melt-quenched GST phase change memory cells gave much lower values: one estimate was $<0.1 \mathrm{~m} / \mathrm{s}$ at $580 \mathrm{~K}$ [38], and Sebastian et al. [39] found even lower values when extrapolated to $600 \mathrm{~K}$.

\section{DISCUSSION AND CONCLUSIONS}

The crystallization of supercooled liquid antimony has been investigated using six DF/MD simulations with three scenarios. The starting configuration of the first (at $600 \mathrm{~K}$ ) was completely disordered and showed no signs of crystallization even after 570 ps; all monitored quantities (MSD, total energy, bond angle distribution, etc.) changed very little during the simulation, indicating that still longer simulations will be needed to obtain spontaneous crystallization via nucleation in such samples at $600 \mathrm{~K}$. The recent dynamic TEM measurements of crystallization [35] showed that nucleation was extremely low in $\mathrm{GeSb}_{6} \mathrm{Te}$, even at the part of the specimen where nucleation was most rapid, indicating that crystallization of $\mathrm{Sb}$ may indeed only occur in the presence of heterogeneities, such as a template or a seed.

Four simulations (runs 0-3) were performed with a crystalline slab template, and one with a fixed crystalline seed. Crystallization occurred in runs $0-3$ in $26-46$ ps, and layerby-layer growth was observed with an average speed of $36 \mathrm{~m} / \mathrm{s}$. The template clearly has a dramatic effect. The sample with the fixed seed required over 140 ps to crystallize completely, with $80-100$ ps being needed to reach the critical nucleus size ( $\sim 60$ atoms, diameter $\sim 11 \AA$ ). The seed grew mainly in a direction perpendicular to the crystalline zigzag layers, and the average crystallization speed after reaching the critical size was $\sim 21 \mathrm{~m} / \mathrm{s}$, about $70 \%$ of the value in run $0-3$. Percolation set in early in the $z$ direction and played an important role, in particular during the fast phase of crystallization. The cubic phases of Sb seen by Hegedüs and Elliott [10] in the early stages of a simulation with an $A 7$ template were not observed, although the sizes and shapes of the simulation cells differ from ours.

Our work confirms that crystallization of fully disordered $\mathrm{Sb}$ is very difficult to simulate above $600 \mathrm{~K}$, but it occurs readily in the presence of a template or seed. The process is faster in $\mathrm{Sb}$ with a template than in our earlier calculations on GST, and the calculated growth rates agree reasonably well with measurements and other calculations. It should be noted, however, that the results of simulations depend on the cell size and shape, and the analysis of experimental data always involves assumptions about the process (classical nucleation theory, presence or absence of Stokes-Einstein behavior, etc.).

Measurements of the fast growth mode of Sb films with small amounts of Ge support the bond-interchange model 
proposed for crystallization in AIST [7]. The present simulations provide more evidence in favor of this simple model, which appears to capture the essence of crystallization in Sb. Diffusion is evident, but it is less important than bond interchange. Finally, cavities (or interstitial space) play a less important role in crystallization of Sb than in GST, with the concentration increasing from $23 \%$ in the liquid phase to $30 \%$ in the crystal (with the short cutoff of $2.6 \AA$ ).

\section{ACKNOWLEDGMENTS}

We acknowledge gratefully the computer time provided by the JARA/HPC partition of the supercomputer JUQUEEN at Forschungszentrum Jülich and for time granted on the supercomputer JURECA at the FZ Jülich. M.R. and J.A. acknowledge financial assistance from the Academy of Finland through its Centres of Excellence Program (Project No. 284621).
[1] E. Bekier, Z. Anorg. Chem. 78, 178 (1912).

[2] N. A. Blum and C. Feldman, Solid State Commun. 15, 965 (1974).

[3] N. Kaiser, Thin Solid Films 116, 259 (1984).

[4] H. Hendus, Z. Phys. 119, 265 (1942).

[5] F. M. Aymerich and A. Delunas, Phys. Status Solidi A 31, 165 (1975).

[6] J. Akola and R. O. Jones, Phys. Status Solidi B 249, 1851 (2012).

[7] T. Matsunaga, J. Akola, S. Kohara, T. Honma, K. Kobayashi, E. Ikenaga, R. O. Jones, N. Yamada, M. Takata, and R. Kojima, Nat. Mater. 10, 129 (2011).

[8] J. Kalikka, J. Akola, and R. O. Jones, Phys. Rev. B 90, 184109 (2014).

[9] G. Eising, B. J. Niebuur, A. Pauza, and B. J. Kooi, Adv. Funct. Mater. 24, 1687 (2014).

[10] J. Hegedüs and S. R. Elliott, Phys. Status Solidi A 207, 510 (2010).

[11] M. Hashimoto, Thin Solid Films 116, 373 (1984).

[12] M. Hashimoto, H. Sugibuchi, and K. Kambe, Thin Solid Films 98, 197 (1982).

[13] A. Kinbara, M. Ohmura, and A. Kikuchi, Thin Solid Films 34, 37 (1976).

[14] M. Hashimoto, T. Niizeki, and K. Kambe, Jpn. J. Appl. Phys. 19, 21 (1980).

[15] R. O. Jones, O. Ahlstedt, J. Akola, and M. Ropo, J. Chem. Phys. 146, 194502 (2017).

[16] J. Akola and R. O. Jones, Phys. Rev. B 85, 134103 (2012).

[17] M. Ropo, J. Akola, and R. O. Jones, J. Chem. Phys. 145, 184502 (2016).

[18] R. O. Jones, Rev. Mod. Phys. 87, 897 (2015).

[19] CPMD V3.15 (C IBM Corp 1990-2011, (C MPI für Festkörperforschung Stuttgart 1997-2001.

[20] N. Troullier and J. L. Martins, Phys. Rev. B 43, 1993 (1991).

[21] J. P. Perdew, A. Ruzsinszky, G. I. Csonka, O. A. Vydrov, G. E. Scuseria, L. A. Constantin, X. Zhou, and K. Burke, Phys. Rev. Lett. 100, 136406 (2008).
[22] S. Nosé, J. Chem. Phys. 81, 511 (1984).

[23] W. G. Hoover, Phys. Rev. A 31, 1695 (1985).

[24] M. J. Assael, A. E. Kalyva, K. D. Antoniadis, R. M. Banish, I. Egry, J. Wu, E. Kaschnitz, and W. A. Wakeham, High Temp. High Press. 41, 161 (2012).

[25] I. Ronneberger, Dr. rer. nat. thesis, RWTH Aachen University, 2016.

[26] J. Kalikka, J. Akola, and R. O. Jones, Phys. Rev. B 94, 134105 (2016).

[27] J. Kalikka, J. Akola, J. Larrucea, and R. O. Jones, Phys. Rev. B 86, 144113 (2012).

[28] B. Chen, J. Momand, P. A. Vermeulen, and B. J. Kooi, Cryst. Growth Des. 16, 242 (2016).

[29] M. Salinga, E. Carria, A. Kaldenbach, M. Bornhöfft, J. Benke, J. Mayer, and M. Wuttig, Nat. Commun. 4, 2371 (2013).

[30] S. L. Roux and V. Petkov, J. Appl. Crystallogr. 43, 181 (2010).

[31] L. Guttman, J. Non-Cryst. Solids 116, 145 (1990).

[32] I. Heimbach, F. Rhiem, F. Beule, D. Knodt, J. Heinen, and R. O. Jones, J. Comput. Chem. 38, 389 (2017).

[33] W. Zhang, I. Ronneberger, P. Zalden, M. Xu, M. Salinga, M. Wuttig, and R. Mazzarello, Sci. Rep. 4, 6529 (2014).

[34] D. J. Adelerhof, Media development for DVD+RW phase change recording, 2004, presented at E/PCOS Conference, Balzers, Liechtenstein, 7-9 September 2004.

[35] M. M. Winseck, H.-Y. Cheng, G. H. Campbell, and M. K. Santala, Dalton Trans. 45, 9988 (2016).

[36] J. Orava, A. L. Greer, B. Gholipour, D. W. Hewak, and C. E. Smith, Nat. Mater. 11, 279 (2012).

[37] I. Ronneberger, W. Zhang, H. Eshet, and R. Mazzarello, Adv. Funct. Mater. 25, 6407 (2015).

[38] R. Jeyasingh, S. W. Fong, J. Lee, Z. Li, K.-W. Chang, D. Mantegazza, M. Asheghi, K. E. Goodson, and H.-S. P. Wong, Nano Lett. 14, 3419 (2014).

[39] A. Sebastian, M. Le Gallo, and D. Krebs, Nat. Commun. 5, 4314 (2014). 